\title{
Classroom reconstruction of the Schwarzschild metric
}

\author{
Klaus Kassner \\ Institut für Theoretische Physik, \\ Otto-von-Guericke-Universität Magdeburg, Germany *
}

(Dated: 11 September 2015)

\begin{abstract}
A promising way to introduce general relativity in the classroom is to study the physical implications of certain given metrics, such as the Schwarzschild one. This involves lower mathematical expenditure than an approach focusing on differential geometry in its full glory and permits to emphasize physical aspects before attacking the field equations. Even so, in terms of motivation, lacking justification of the metric employed may pose an obstacle. The paper discusses how to establish the weak-field limit of the Schwarzschild metric with a minimum of relatively simple physical assumptions, avoiding the field equations but admitting the determination of a single parameter from experiment. An attractive experimental candidate is the measurement of the perihelion precession of Mercury, because the result was already known before the completion of general relativity. It is shown how to determine the temporal and radial coefficients of the Schwarzschild metric to sufficiently high accuracy to obtain quantitative predictions for all the remaining classical tests of general relativity.
\end{abstract}

PACS numbers: 01.40.gb; 04.20.-q; 04.20.Cv; 04.80.Cc

Keywords: Physics education, general relativity, Schwarzschild metric

\section{INTRODUCTION}

Conceptually speaking, general relativity (GR) is not a particularly difficult theory. From the viewpoint of physics education, all the conceptual impositions of the relativity theories arguably arise with special relativity (SR) already. It is in courses of SR that students will be exposed to the relativity of simultaneity and a variety of paradoxes, having to do with time dilation and differential aging, 1,2 length contraction and the pole-barn paradox,,$\frac{3}{b}$ bridges that may or may not collapse under rel-

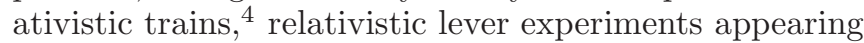
to violate angular momentum conservation, $\underline{\underline{5}}$ Bell's spaceship paradox,$\frac{6.7}{2}$ Ehrenfest's paradox ${ }^{8-10}$ and the appearance of non-Euclidean geometry in accelerating systems such as a rotating disk $10-12$ Those students will probably not be overly shocked by the additional complication of spacetime curvature in GR.

And this is essentially the only conceptual complication. Some things even get simpler with certain standard examples of GR systems. In SR, we have the bewildering phenomenon of mutual time dilation, utterly incomprehensible without an understanding of the nonabsoluteness of simultaneity. When comparing coordinate stationary observers (CSOs) in a static metric, there usually also is time dilation, but different observers agree on whose clocks are runnig faster, a situation that is not especially difficult to visualize.

What makes GR difficult, is the mathematical overhead. SR can be taught with very little calculus, whereas in GR, differential geometry is essential. The field equations of GR are intrinsically nonlinear, so their solution is, even in the simplest cases, nontrivial. The Riemann curvature tensor has 20 independent components.

Given the conceptual simplicity and the mathematical complexity of GR, it is natural to ask whether it is possible to find a simpler approach to certain fundamental aspects of the theory, to make it more accessible to students in the transition from special to general relativity.
A full-fledged course in GR will have to deal with the field equations eventually, but the entry point into the theory might be based on much simpler considerations. An SR course giving a glimpse at GR near its end may benefit from avoiding the field equations altogether.

It is a substantiated view that exploring the consequences of a particular metric (normally the Schwarzschild one) leads to an accessible "physics first" approach to introducing GR.13,14 Unfortunately, the metric will arise out of the blue in such a strategy. Therefore, it is legitimate to inquire whether we can do better and obtain nontrivial metrics from simple arguments, without going all the way to the field equations.

Such an idea was implicit in the so-called Lenz-Schiff argument, apparently never published by Lenz but presented in Sommerfeld's textbook ${ }^{15}$ and used by Schiff ${ }^{16}$ to argue that light deflection by the sun is quantitatively describable without the field equations. This would put it on a par with the gravitational redshift of spectral lines in the field of a weakly gravitating object such as our sun, known to be explicable by a combination of special relativity with the Newtonian limit (NL), using Einstein's equivalence principle (EP). The perihelion precession of Mercury would then remain the only one of the three classical tests of GR that really probes the field equations.

Schiff's paper was shown to be in error ${ }^{17.18}$ Nevertheless, recurrently ${ }^{19}$ and even recently ${ }^{20}$ articles have been published that "derive" GR effects requiring spacetime curvature on the basis of the fallacious Lenz-Schiff argument. Yet, detailed arguments had been given ${ }^{21,22}$ that a simple derivation of the Schwarzschild metric, i.e., one avoiding knowledge that traditionally is gathered from the field equations, is impossible.

The appearance of a controversy may be deceiving. Advocates of the Lenz-Schiff argument seem to be unaware of its deficiencies. In contrast, anyone familiar with the foundations of GR will realize that any nonsingular metric (with Minkowskian signature) is locally compati- 
ble with SR, due to the equivalence principle (stating that it always possible to transform the metric to Minkowski form locally). Therefore, the EP does not constrain the metric. Without the field equations or some equivalent, constraints on the metric arise only from symmetry and the NL. Any corrections to the NL, expressible in powers of the small quantity $G M / c^{2} r$ for a spherically symmetric situation, ${ }^{23}$ must be missed by a Lenz-Schiff type approach as, in fact, by any other approach based on local considerations within the framework of SR. Therefore, no more than a weak-field approximation to the metric can be gained. This does not yet exclude Schiff's result, essentially referring to the first-order term in powers of $G M / c^{2} r$ of the radial metric coefficient $g_{r r}$. However, the aforementioned analyses 21,22 show that the Newtonian limit gives us only $g_{r r}=1$. Thereafter, any claims to deriving the Schwarzschild metric or even a post-Newtonian approximation to it on the basis of just symmetry, SR, the EP, and the NL, are recognizably erroneous.

To be precise, this does not mean that one cannot do without the field equations.

Essentially, either the field equations or their generating action including its Einstein-Hilbert part are a set of postulates within GR. In most theories based on postulates or axioms, the axioms are not unique. In set theory, for example, the axiom of choice, Zorn's Lemma and the well-ordering theorem are all interchangeable ${ }^{24}$ It is sufficient to postulate one of them. The other two are then derivable. In thermodynamics, there are various different formulations of the second law, which is a postulate of the theory. It is sufficient to take one of them, then the others can be derived as theorems. ${ }^{25}$

If consideration is restricted to the static spherically symmetric case, it may be possible to use a simpler postulate (or two) than the one leading to the field equations to derive a metric. Due to the restriction to spherical geometry, there is no need that the postulate be powerful enough to replace the field equations altogether. It is sufficient, if it can replace them in spherically symmetric situations 26 This kind of approach is not only logically possible, it has even been discussed favorably by Sacks and Ball 17 with regard to Tangherlini's postulational approach to the Schwarzschild metric 27 Unfortunately, Rindler later showed one of the two Tangherlini postulates to be unconvincing. ${ }^{28}$ But clearly, Tangherlini's approach is not subject to the criticism (nor the impossibility proof) offered by Gruber et $a l_{.}^{22}$

Since both postulates from Ref. 27 cannot be used here, the exact Schwarzschild metric will not be obtained. However, I use one argument beyond the aforementioned ingredients (symmetry, SR, EP, NL) to restrict the form of the metric. This will reduce to the more convinicing one of Tangherlini's two postulates, and it will be better justified than his statement. On the other hand, additional information will be needed to obtain a truly postNewtonian approximation, and this can be taken from experiment. Amusingly, all of this information was available in 1911, when Einstein published a calculation of light deflection by the sun, ${ }^{29}$ reproducing von Soldner's century-old result ${ }^{30}$ and thus missing the correct prediction by a factor of 2 .
The general outline of the paper is as follows. In Sec.II, the metric describing the closest approximation to a uniform gravitational field that is relativistically possible, also known as the Rindler metric, is derived. This simple problem, rigorously solvable within special relativity, serves to expose the interplay of symmetry arguments and thought experiments allowing us to obtain metric coefficients, an approach that then may be applied to more complex situations. It also demonstrates a littleappreciated property of the Rindler metric justifying its interpretation as describing a uniform gravitational field. Section III is devoted to an introductory attempt at constructing the metric of a spherical mass distribution using symmetry, the EP and the NL. At first sight, this approach succeeds in obtaining the exact Schwarzschild metric. Its deficiencies become visible on analysis of the order of approximation achieved. A plausible and simple physical assumption turns out to partially cure the problem. In Sec. IV it will be shown how information from a true experiment, 31 viz. measurement of the perihelion precession of Mercury, may then be used to resolve the issue and to obtain the weak-field limit of the Schwarzschild metric with sufficient accuracy to quantitatively predict light deflection by the sun, as demonstrated in Sec. D] and the Shapiro delay $\underline{32}$ That section also discusses how the aforementioned factor of 2 can be found without actually performing the full calculation. Finally, some conclusions are given in Sec. VI.

Most of the material is presented as if we did not know GR yet, but occasionally this stratagem is dropped to address teachers directly, who are assumed to be more knowledgeable. We shall assume general acquaintance with SR and the use of different coordinate representations of the Minkowski metric.

\section{DERIVATION OF THE METRIC OF A "UNIFORM" GRAVITATIONAL FIELD}

Our first aim is to deal with the inertial field inside an accelerating object, say a big spacecraft. This situation may be completely described within SR. At each point, the acceleration is to be constant in time, i.e., each observer feels a constant proper acceleration. In Newtonian physics, we would get something resembling a uniform gravitational field, if all observers had the same acceleration. In relativistic physics, we know that if observers arranged along the direction of acceleration had the same proper acceleration, Bell's spaceship paradox 6.7 would apply - they would find each other moving apart. Rather, we are interested in a situation that is considered static by all observers. This is Born rigid motion, 33 in which the proper distances between our aligned observers remain constant, which means that from the vantage point of an inertial system leading observers must accelerate more slowly than trailing ones, so their distance shrinks precisely according to the appropriate Lorentz factor.

Let us introduce an inertial frame $\Sigma$ with time $T$ and cartesian coordinates $X, Y, Z$ and have its $X$ axis oriented parallel to the direction of acceleration. Consider first a single (point-like) observer $O$. Since his proper ac- 
celeration is constant, he will feel a constant accelerating force $f_{0}$, which is also the force, by which a momentarily comoving inertial observer (in frame $\Sigma^{\prime}$ ) will perceive $O$ to be accelerated. Moreover, the relativistic transformation law for forces parallel to the vector of relative motion between inertial systems tells us that the force $F_{0}$, by which $O$ is accelerated in $\Sigma$ is the same as in $\Sigma^{\prime}$ : $F_{0}=f_{0}$. Then the equation of motion for $O$ 's trajectory in $\Sigma$ reads

$$
\frac{\mathrm{d}}{\mathrm{d} T} m \gamma(V) V=f_{0}
$$

where $V(T)=\mathrm{d} X / \mathrm{d} T$ and $\gamma(V)=1 / \sqrt{1-V^{2} / c^{2}}$. If we set the time $T$ equal to zero at the moment when $V=0$, this is solved by

$$
\gamma(V) V=a T
$$

where $a=f_{0} / m$ is the proper acceleration of $O$. Solving for $V$, we have

$$
V=\frac{a T}{\sqrt{1+\left(\frac{a T}{c}\right)^{2}}} \Rightarrow \gamma(V)=\sqrt{1+\left(\frac{a T}{c}\right)^{2}}
$$

and this can be integrated once more to obtain

$$
X=\frac{c^{2}}{a}\left(\sqrt{1+\left(\frac{a T}{c}\right)^{2}}-1\right)+X_{0} .
$$

The trajectory $X(T)$ is a hyperbola rather than the parabola known from Newtonian physics, hence the notion of hyperbolic motion. Note that by taking the time derivative in (3), we end up with the standard relationship for the transformation of longitudinal acceleration: $A \equiv \mathrm{d}^{2} X / \mathrm{d} T^{2}=a / \gamma(V)^{3}$. Next, consider two observers $O_{1}$ and $O_{2}$, starting at $X_{01}$ and $X_{02}$ with proper accelerations $a_{1}$ and $a_{2}$, so their trajectories are given by

$$
\begin{aligned}
& X_{1}=\frac{c^{2}}{a_{1}}\left(\sqrt{1+\left(\frac{a_{1} T}{c}\right)^{2}}-1\right)+X_{01}, \\
& X_{2}=\frac{c^{2}}{a_{2}}\left(\sqrt{1+\left(\frac{a_{2} T}{c}\right)^{2}}-1\right)+X_{02},
\end{aligned}
$$

and require the distance between them to remain constant in the frame of the first $\underline{34}$ The Lorentz transformations from $\Sigma$ to an inertial frame momentarily comoving with $O_{1}$ read

$$
\begin{aligned}
& x=\gamma\left(X-X_{1}-V\left(T-T_{1}\right)\right), \quad \gamma=\sqrt{1+\left(\frac{a_{1} T_{1}}{c}\right)^{2}}, \\
& t=\gamma\left(T-T_{1}-\frac{V}{c^{2}}\left(X-X_{1}\right)\right)
\end{aligned}
$$

and they transform the point $\left(T_{1}, X_{1}\right)$ to the origin of the comoving inertial observer, in whose frame the distance $D$ between $O_{1}$ and $O_{2}$ at this moment is obtained by setting $X=X_{2}$ and choosing $T=T_{2}$ so that $t=0$. This gives

$$
\begin{aligned}
& T_{2}=T_{1}+\frac{V}{c^{2}}\left(X_{2}-X_{1}\right), \\
& D=x_{2}=\left(X_{2}-X_{1}\right) / \gamma \Rightarrow T_{2}=T_{1}+\frac{\gamma(V) V}{c^{2}} D .
\end{aligned}
$$

Requiring in addition that the velocity of $\mathrm{O}_{2}$ with respect to $O_{1}$ be zero, we find $\left.\frac{\mathrm{d} X}{\mathrm{~d} T}\right|_{X=X_{2}}=V$ (obviously). This implies $a_{1} T_{1}=a_{2} T_{2}$, which together with (2) and (7) leads to $a_{1} T_{1} / a_{2}=T_{1}\left(1+a_{1} D / c^{2}\right)$. We then obtain the important relationship

$$
D=\frac{c^{2}}{a_{2}}-\frac{c^{2}}{a_{1}}=X_{02}-X_{01} .
$$

Thus, by choice of the origin of $\Sigma$, we may achieve $X_{0 i}=c^{2} / a_{i}$ for observer $O_{i}$ where originally $i=1,2$, but evidently, this can be extended to an arbitrary number of observers. Equation (44) for the trajectory of an observer starting from $X_{0}=x$ then simplifies to

$$
X=\sqrt{x^{2}+c^{2} T^{2}} .
$$

If we fill a half-space with observers labeled by their positive initial coordinate $x$ and have them move according to (9) with their $Y$ and $Z$ coordinates unchanged, the ensemble will perform Born rigid motion.

To obtain the metric describing the common rest frame of these observers, we note that translational symmetry in the $y$ and $z$ directions as well as the requirement of time independence of the metric imply the following general form of the spacetime line element ${ }^{35}$

$$
\mathrm{d} s^{2}=-F(\tilde{x}) c^{2} \mathrm{~d} t^{2}+G(\tilde{x}) \mathrm{d} \tilde{x}^{2}+\mathrm{d} y^{2}+\mathrm{d} z^{2} .
$$

Here, we have temporarily garnished one coordinate with a tilde, because for $G(\tilde{x}) \neq 1$, the proper length element $\mathrm{d} \ell$ of this metric $\left(\mathrm{d} \ell^{2}=\left.\mathrm{d} s^{2}\right|_{\mathrm{d} t=0}\right.$, due to timeorthogonality) does not have its standard form, whereas our relationship for the proper acceleration derived above was formulated in terms of the proper distance. The simple coordinate transformation

$$
x(\tilde{x})=\int^{\tilde{x}} \sqrt{G(u)} \mathrm{d} u
$$

turns (10) into

$$
\mathrm{d} s^{2}=-f(x) c^{2} \mathrm{~d} t^{2}+\mathrm{d} x^{2}+\mathrm{d} y^{2}+\mathrm{d} z^{2},
$$

so all that remains to be determined is the function $f(x)$.

The proper time of a CSO, i.e., an observer satisfying $\mathrm{d} x=\mathrm{d} y=\mathrm{d} z=0$, is given by $\left(\mathrm{d} s^{2}=-c^{2} \mathrm{~d} \tau^{2}\right)$

$$
\mathrm{d} \tau=\sqrt{f(x)} \mathrm{d} t
$$

so observers at positions $x_{1}$ and $x_{2}$ will find their standard clocks be subject to time dilation according to

$$
\frac{\mathrm{d} \tau_{2}}{\mathrm{~d} \tau_{1}}=\sqrt{\frac{f\left(x_{2}\right)}{f\left(x_{1}\right)}}
$$


To determine the time dilation factor, imagine that $O_{1}$ sends an electromagnetic signal having the frequency $\nu_{1}$ to the very close $\mathrm{O}_{2}$, who will receive it at frequency $\nu_{2}$. A good inertial frame to discuss this in is the frame momentarily comoving with $O_{1}$ at the emission event. During the short time interval $\Delta \tau=\left(x_{2}-x_{1}\right) / c$ taken by the light, $\mathrm{O}_{2}$ will have picked up the small velocity $v=a\left(x_{2}\right) \Delta \tau$ in this inertial frame. If $x_{2}>x_{1}, O_{2}$ is moving away from the emission event (since both the signal and $\mathrm{O}_{2}$ are moving towards increasing $x$ ), so the frequency $\nu_{2}$ on reception of the signal will be lower than $\nu_{1}$, due to the Doppler effect. The relativistic Doppler shift is given by

$$
\frac{\nu_{1}}{\nu_{2}}=\sqrt{\frac{c+v}{c-v}} \approx 1+\frac{v}{c} \approx 1+\frac{a\left(x_{2}\right) \Delta \tau}{c} \approx 1+\frac{a\left(x_{2}\right) \Delta x}{c^{2}} .
$$

We introduce an acceleration potential via $\mathrm{d} \Phi=a(x) \mathrm{d} x$. Then (14) and (15) imply $\left(\mathrm{d} \tau_{2} / \mathrm{d} \tau_{1}=\nu_{1} / \nu_{2}\right)$, for sufficiently small $x_{2}-x_{1}$ :

$$
\sqrt{\frac{f\left(x_{2}\right)}{f\left(x_{1}\right)}}=1+\frac{\Phi\left(x_{2}\right)-\Phi\left(x_{1}\right)}{c^{2}}
$$

which can be easily converted into a differential equation for $f(x)$,

$$
\begin{aligned}
\sqrt{\frac{f(x+\mathrm{d} x)-f(x)}{f(x)}+1} & =1+\frac{1}{2} \frac{f^{\prime}(x)}{f(x)} \mathrm{d} x \\
& =1+\frac{1}{c^{2}} \Phi^{\prime}(x) \mathrm{d} x, \\
\frac{1}{2} \frac{f^{\prime}(x)}{f(x)} & =\frac{1}{c^{2}} \Phi^{\prime}(x),
\end{aligned}
$$

and this is solved by

$$
f(x)=\mathrm{e}^{2 \Phi / c^{2}}
$$

where the integration constant can be chosen by fixing an additive constant implicit in the definition of $\Phi(x)$. Since we know the position dependence of the proper acceleration $a(x)$, it is straightforward to obtain the potential:

$$
\frac{\mathrm{d} \Phi}{\mathrm{d} x}=a(x)=\frac{c^{2}}{x} \Rightarrow \Phi(x)=c^{2} \ln x+\text { const. }
$$

This gives

$$
f(x)=\frac{g^{2} x^{2}}{c^{4}}
$$

where $g$ is the proper acceleration at the position $x_{0}$, where $f\left(x_{0}\right)=1$.

Even though we have now successfully derived the Rindler metric

$$
\mathrm{d} s^{2}=-\frac{g^{2} x^{2}}{c^{4}} c^{2} \mathrm{~d} t^{2}+\mathrm{d} x^{2}+\mathrm{d} y^{2}+\mathrm{d} z^{2},
$$

it is useful to look at another thought experiment. Consider an observer at $x_{0}$ who slowly lowers some small mass $m$, hanging from an inextensible massless tether, towards smaller $x$ values.

Of course, in relativity, there are no truly inextensible bodies, because they would allow infinitely fast signaling (pull at one end to immediately transfer a message to the other). However, we do not need more than approximate inextensibility. An inextensible tether is simply one with a very large Young's modulus $Y$. The larger we make $Y$, the better the approximation. The kind of inextensibility we want here may be expressed in terms of the covariant formulation of Hooke's law: ${ }^{36}$ All length changes required by relativistic kinematics are allowed, but local proper length increments of the tether do not change under a force. $\frac{37}{2}$ The only reason for this requirement is to avoid the consideration of elastic or plastic effects. Since we use our tether for quasistatic transport only, fast signaling will not occur 38

What is important in the following is that if a piece $\mathrm{d} \ell$ of the tether is threaded down at its upper end, the lower end will move down by the same amount $\mathrm{d} \ell$ in terms of its local proper length. The question we ask then is: what is the force needed to hold the mass at position $x$ ? At the beginning of the process, i.e., at $x_{0}$, we clearly expect the force to be $F=-m g$, but as the mass is lowered, it will experience different local proper accelerations. A way to calculate the force is to invoke energy conservation. On being lowered, the mass is doing work, so we should have

$$
F=-\frac{\mathrm{d} E(x)}{\mathrm{d} \ell}=-\frac{\mathrm{d} E(x)}{\mathrm{d} x}
$$

where $E(x)$ is its energy at position $x$, as judged by the observer at $x_{0}$. Now locally, the mass always has energy $m c^{2}$, as it does not acquire kinetic energy - the experiment is performed quasistatically. But the observer at $x_{0}$ will not assign this local value to energy, because to him everything at $x$ happens at a slower rate due to time dilation. This reduces the energy of photons by the time dilation factor. Clearly, all other energies must be affected the same way, otherwise no consistent physical description would be possible. To see this in more detail, imagine that the energy of massive particles is reduced by a factor $\beta<1$, that of photons only by a factor $\alpha>\beta$. Suppose the local observer has an electron and a positron annihilate to produce two photons, the energy of which locally is given by the sum of the particle energies: $2 h \nu=E_{e}+E_{e^{+}}$. For the distant observer, energy conservation would be violated, because the energy of the two photons would be $2 \alpha h \nu$, that of the particles $\beta\left(E_{e}+E_{e^{+}}\right)$, and $2 \alpha h \nu>\beta\left(E_{e}+E_{e^{+}}\right)$. By an appropriate procedure, with the lower observer sending photons to the upper one, who converts them into particles that he sends down, where they are converted into photons again, a perpetuum mobile (of the first kind!) could be built 39

A perpetuum mobile could of course also be constructed, if $\alpha<\beta$, using the reversed sequence of processes.

From these considerations we conclude

$$
E(x)=\sqrt{\frac{f(x)}{f\left(x_{0}\right)}} m c^{2}=\sqrt{f(x)} m c^{2},
$$


(because $f\left(x_{0}\right)=1$ ), hence

$$
F(x)=-m c^{2} \frac{f^{\prime}(x)}{2 \sqrt{f(x)}},
$$

which evaluates to $F=-m g$. Therefore, the force exerted by a mass hanging from a tether is constant for

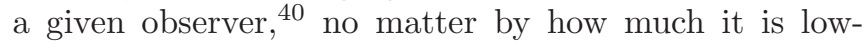
ered in the "inertial field", a fact that has been noted by Grøn before $\underline{\underline{41}}$ This is the meaning of "uniform" when we are talking about the uniform gravitational field - homogeneity of the force on a particle or an object in a fixed observer's frame rather than homogeneity of acceleration (i.e., homogeneity of the force per unit mass). A detailed discussion of the issue of uniformity of fields in GR is given in Ref. 42 .

Note that we could have derived the metric by requiring the tether force to be constant and using (24). This derivation would be less rigorous than the one actually given but it would be physically well motivated and the result would be valid.

The Rindler metric is related to the Minkowski metric by a coordinate transformation

$$
c T=x \sinh \frac{g}{c} t, \quad X=x \cosh \frac{g}{c} t, \quad Y=y, \quad Z=z,
$$

so it describes a flat spacetime, the curvature of which necessarily vanishes. In modern parlance, gravity is often identified with the curvature of spacetime, but Einstein's view rather was that inertial and gravitational fields are identical in nature. Moreover, while the equivalence principle declares this identity only locally for inhomogeneous gravitational fields, it is not inconceivable that a mass distribution (homogeneous in $y$ and $z$ ) could be constructed theoretically that would produce the metric (21) in the vacuum delimiting it above some $x$ value. Would we then refuse to call the corresponding attractive field gravitational just because spacetime happens not to be curved?

\section{THE METRIC OUTSIDE A SPHERICAL MASS DISTRIBUTION}

Next, we would like to extend the ideas developed so far to a nontrivial gravitational field, one that cannot be obtained by a simple coordinate transformation from the Minkowski metric.

A leading theme in general relativity is to explain gravity in terms of spacetime geometry; in particular, the notion of curvature of spacetime becomes important. A basic object in describing spacetime geometry is the metric. There are more complex objects, derivable from the metric (such as the Riemann curvature tensor), that may be used to decide whether the metric describes a flat or a curved spacetime. None of these objects will be needed here. It may be sufficient to say that if we write done some metric randomly, it is much more likely that it will describe a curved spacetime than not. A condition for flatness is that there exists a global coordinate transformation that takes our metric to Minkowski form. Since this is true only when certain integrability conditions are met (that are expressible via the Riemann tensor), most metrics that we may care to write down will comprise curvature and this is related to the presence of gravitation according to GR.

One of the simplest gravitating systems is a time-independent spherically symmetric mass distribution. We expect it to be describable by a static spherically symmetric metric. As we shall convince ourselves presently, the line element may then, without loss of generality, be written as

$$
\mathrm{d} s^{2}=-f(r) c^{2} \mathrm{~d} t^{2}+g(r) \mathrm{d} r^{2}+r^{2}\left(\mathrm{~d} \vartheta^{2}+\sin ^{2} \vartheta \mathrm{d} \varphi^{2}\right) .
$$

Here, $\vartheta$ and $\varphi$ are the usual angular coordinates which, due to spherical symmetry, may only appear in the combination $\mathrm{d} \Omega^{2}=\mathrm{d} \vartheta^{2}+\sin ^{2} \vartheta \mathrm{d} \varphi^{2}$ but not in any of the coefficient functions. Because the metric is assumed time independent, none of the coefficients may depend on $t$. Therefore, all of them may be functions of the radial coordinate $r$ only. The prefactor of $\mathrm{d} \Omega^{2}$ might contain some additional function of $r$, which we can however get rid of by redefining $r$ so that the surface of any sphere about the coordinate center, given by $r=$ const., becomes $4 \pi r^{2}$. Finally, a term of the form $h(r) \mathrm{d} t \mathrm{~d} r$ would be allowed by symmetry, but can be removed by a coordinate transformation $t \rightarrow t+w(r) \underline{43}$

At large radii, gravitation will become negligible, so the metric should approach Minkowskian form, hence we require

$$
\lim _{r \rightarrow \infty} f(r)=1, \quad \lim _{r \rightarrow \infty} g(r)=1 .
$$

So far, we have not used any physics, just symmetry. In order to determine $f(r)$ and $g(r)$, we need to invoke physical ideas.

First, we make use of the equivalence principle. Instead of translating the physics in an accelerating system into terms of a gravitating one, which requires to visualize two different but equivalent systems in parallel, let us consider a freely falling observer in the actual system under consideration. $\underline{44}$ The prescription then is to describe local physics in the frame of that inertial observer by SR. For the freely falling observer, there is no gravitational field and everything that the gravitational field does to CSOs must be due to the fact that they are accelerating with respect to his inertial frame. Note that an infinity of freely falling observers may be chosen at any point. Normally, the best choice is to consider one that is momentarily at rest with respect to the object (e.g., a CSO) that is to be described.

Using the EP, we obtain a relationship between the two functions to be determined and the local gravitational acceleration. Consider two very close CSOs $A$ at $r_{1}$ and $B$ at (the same angular position at) $r_{2}$ with $r_{2}>r_{1}$, plus an inertial observer $C$ momentarily comoving with $A$, the moment $A$ sends a light signal to $B$, as depicted in Fig. 1

In $C$ 's frame, the frequency $\nu_{1}$ of the signal is unchanged during its short transit time $\Delta \tau$. But $B$, having 


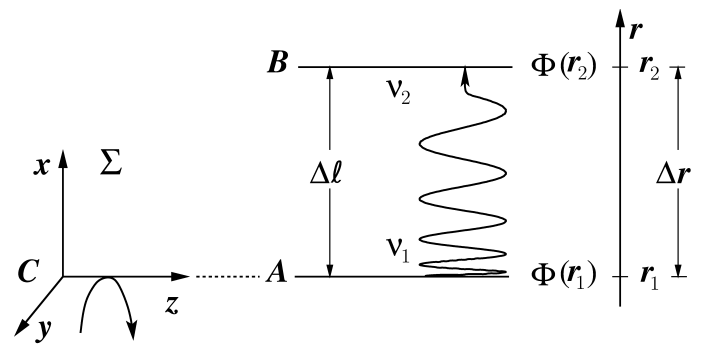

FIG. 1. Equivalence principle: The inertial observer $C$ arrives at the apex of his trajectory next to $A$ the moment $A$ sends off a signal to $B$.

accelerated to a small velocity $v=a\left(r_{2}\right) \Delta \tau$, will receive it at a reduced Doppler shifted frequency $\nu_{2}$. The deviation of the ratio of frequencies from 1 is attributed to time dilation by $A$ and $B$, who are stationary. From $\mathrm{d} s^{2}=-c^{2} \mathrm{~d} \tau^{2}$, we read off that the proper time of a CSO is given by $\mathrm{d} \tau=\sqrt{f(r)} \mathrm{d} t$, so the frequency ratio may be calculated as

$$
\frac{\nu_{1}}{\nu_{2}}=\frac{\mathrm{d} \tau_{2}}{\mathrm{~d} \tau_{1}}=\frac{\sqrt{f\left(r_{2}\right)}}{\sqrt{f\left(r_{1}\right)}} .
$$

For $C$, the special relativistic Doppler shift formula yields

$$
\frac{\nu_{1}}{\nu_{2}}=\sqrt{\frac{c+v}{c-v}} \approx 1+\frac{v}{c} .
$$

Denote by $\Delta \ell$ the local proper distance separating $A$ and $B$. The metric is time orthogonal, therefore $\mathrm{d} \ell^{2}=$ $\left.\mathrm{d} s^{2}\right|_{\mathrm{d} t=0}$. We then have, for the transit time, $\Delta \tau=$ $\Delta \ell / c=\sqrt{g(r)}\left(r_{2}-r_{1}\right) / c$ (with $\left.r \in\left[r_{1}, r_{2}\right]\right)$. Due to the closeness of the three observers and the smallness of their relative motion, the length $\Delta \ell$ is the same for all of them during the sequence of events considered. It is again useful to introduce an acceleration potential, describing local proper acceleration, via $\mathrm{d} \Phi=a(r) \mathrm{d} \ell=a(r) \sqrt{g(r)} \mathrm{d} r$. This provides

$$
\frac{v}{c} \approx \frac{a \Delta \ell}{c^{2}} \approx \frac{\Delta \Phi}{c^{2}}
$$

and, for sufficiently small $\left|r_{2}-r_{1}\right|$

$$
\begin{aligned}
\sqrt{\frac{f\left(r_{2}\right)}{f\left(r_{1}\right)}} & =1+\frac{\Phi\left(r_{2}\right)-\Phi\left(r_{1}\right)}{c^{2}}, \\
a(r) & =\frac{1}{\sqrt{g(r)}} \frac{\mathrm{d} \Phi}{\mathrm{d} r} .
\end{aligned}
$$

The first of these two equations is converted into a differential equation as before

$$
\frac{1}{2} \frac{f^{\prime}(r)}{f(r)}=\frac{1}{c^{2}} \Phi^{\prime}(r)
$$

and this is solved by

$$
f(r)=\mathrm{e}^{2 \Phi / c^{2}},
$$

where the integration constant has been fixed by the boundary condition (27), given that, for large $r, \Phi$ must reduce to the Newtonian potential, i.e., go to zero in the standard gauge. Since we do not know anything about $\Phi(r)$ for small $r$, Eq. (34) means no more in that $r$ range than expressing one unknown function, $f(r)$, in terms of another equally unknown one, $\Phi(r)$. All we achieve by this is to equate $f(r)$ to a quantity that has the physical interpretation of a potential. Nevertheless, the result is useful, as it is this interpretation that allows us to deduce the functional form of $f(r)$ at large $r$ by the requirement that the potential become Newtonian there.

To obtain a second relationship for the two functions $f(r)$ and $g(r)$, let us look at the same thought experiment as in the case of a uniform field. Assume that an observer at $r_{0}$ slowly lowers some mass $m$ hanging from an inextensible massless tether towards smaller $r$ values.

As before, we express the force using energy conservation,

$$
F=-\frac{\mathrm{d} E(r)}{\mathrm{d} \ell}=-\frac{\mathrm{d} E(r)}{\mathrm{d} r} \frac{\mathrm{d} r}{\mathrm{~d} \ell},
$$

where $E(r)$ is the energy of the mass at position $r$, as judged by the observer at $r_{0}$. By the same kind of argument as in the uniform gravitational field we now obtain

$$
E(r)=\sqrt{\frac{f(r)}{f\left(r_{0}\right)}} m c^{2} \underset{r_{0} \rightarrow \infty}{\rightarrow} \sqrt{f(r)} m c^{2},
$$

where for simplicity our observer was moved to infinity. The force felt at the upper end of the tether then is

$$
F=-m c^{2} \frac{\mathrm{d} \sqrt{f(r)}}{\mathrm{d} r} \frac{1}{\sqrt{g(r)}}=-m c^{2} \frac{f^{\prime}(r)}{2 \sqrt{f(r) g(r)}} .
$$

Now we require that for large $r, \Phi(r)$ and $F(r)$ take their Newtonian limits, i.e.,

$$
\begin{aligned}
& \Phi(r)=-\frac{G M}{r}, \\
& F(r)=-\frac{G m M}{r^{2}} .
\end{aligned}
$$

At this moment, we have no idea about what will become of these laws as relativistic effects become strong, so we content ourselves with determining a weak-field limit of the metric. The relevant quantity distinguishing between weak and strong is $\Phi(r) / c^{2}$, which outside the sun does not exceed $10^{-5}$ in our solar system, so this limit should be appropriate for all calculations referring to the latter.

Equations (34) and (37) together with expressions (38) and (39) for the potential and force may be used to determine the two functions $f(r)$ and $g(r)$ :

$$
\begin{aligned}
f(r) & =\mathrm{e}^{2 \Phi / c^{2}} \approx 1+2 \frac{\Phi}{c^{2}}=1-\frac{2 G M}{r c^{2}}, \\
-\frac{G m M}{r^{2}} & =-m \frac{G M}{r^{2}} \frac{1}{\sqrt{f(r) g(r)}} \\
\Rightarrow g(r) & =\frac{1}{f(r)}=\frac{1}{1-\frac{2 G M}{r c^{2}}} .
\end{aligned}
$$


This gives us, as a weak-field approximation, the line element

$$
\begin{aligned}
\mathrm{d} s^{2}= & -\left(1-\frac{2 G M}{r c^{2}}\right) c^{2} \mathrm{~d} t^{2}+\frac{1}{1-\frac{2 G M}{r c^{2}}} \mathrm{~d} r^{2} \\
& +r^{2}\left(\mathrm{~d} \vartheta^{2}+\sin ^{2} \vartheta \mathrm{d} \varphi^{2}\right)
\end{aligned}
$$

which is the exact result for the Schwarzschild metric!

Of course, this is too good to be true. To see what happened, let us use, instead of the last formula from (37), the first. This means, we approximate $\sqrt{f(r)}$ instead of $f(r)$ itself. Then the calculation reads

$$
\begin{aligned}
\sqrt{f(r)} & =\mathrm{e}^{\Phi / c^{2}} \approx 1+\frac{\Phi}{c^{2}}=1-\frac{G M}{r c^{2}}, \\
-\frac{G m M}{r^{2}} & =-m \frac{G M}{r^{2}} \frac{1}{\sqrt{g(r)}} \\
\Rightarrow g(r) & \equiv 1,
\end{aligned}
$$

which is not the expected result for $g(r)$.

In the two calculations, we used approximations for $f(r)$ which agreed to first order in the small quantity $G M / r c^{2}\left(\approx \Phi / c^{2}\right)$, but not to second order. However, the structure of the equations is such that the first-order term of $g(r)$ depends on the second-order term of $f(r)$. This becomes immediately clear, if we plug the approximation

$$
f(r)=1-\frac{2 G M}{r c^{2}}+\beta_{2}\left(\frac{G M}{r c^{2}}\right)^{2}
$$

into (37) with the force law (39). No matter whether we take the formula with the derivative of $f(r)$ or that with the derivative of its square root, we obtain the same first-order result for $g(r)$, providing we expand the square root correctly to second order:

$$
g(r)=1+2\left(1-\beta_{2}\right) \frac{G M}{r c^{2}} .
$$

Our first approximation corresponds to $\beta_{2}=0$, the second to $\beta_{2}=1$.

Therefore, to obtain a nontrivial result for $g(r)$, we need to know $f(r)$ or, since the relationship (34) between $f(r)$ and the potential is exact $\stackrel{45}{r}$ the potential $\Phi(r)$ at least to the next order in $G M / r c^{2}$. Knowing the exact result for $f(r)$ from GR, we may infer that Eq. (38) is indeed only a lowest-oder approximation. $\underline{\underline{46}}$

This immediately begs the question whether this is true for (39) as well. Indeed, if we had to replace the force law by $F(r)=-G m M / r^{2}\left(1+\gamma_{1} G M / r c^{2}+\ldots\right)$, this would bring in another unknown coefficient and diminish our chances of calculating anything meaningful.

There are two reasons to believe - without prior knowledge of the exact result - that (39) is, in fact, exact. The first is that a similar thing happened in the case of the Rindler metric. A force measured with tethers as described turns out to be constant in space just as in a Newtonian uniform gravitational field. That the Newtonian force law be valid beyond the weak-field approximation in the spherically symmetric situation as well was one of the two postulates introduced by Tangherlini: 27
It may be considered an extrapolation from the homogeneous to the inhomogeneous case, hence a suggestive but not really a strong argument. However, there is a much more convincing way to justify this assumption.

The force $F(r)$ is a global field, measurable using tethers $\stackrel{47}{ }$ In principle, the field may be measured in all of space (outside the central star). Calculating the integral of the force over the surface of a sphere of radius $r$, we obtain its total flux through that surface. If we evaluate it on two concentric shells, the integral should not change, if there is no source of the field between the two shells, i.e. in vacuum. In fact, experience with both Newtonian gravity and electrodynamics suggests this quantity to be a fixed multiple of the total "charge" enclosed by the shells (mass, electrical charge) that is at the origin of the field. This charge should be a conserved quantity - in GR we expect it to be related to mass-energy. Therefore, if such a conservation law prevails in GR, the force must be proportional to $1 / r^{2}$ outside the spherically symmetric mass distribution, if the area of the surface of a sphere grows as $r^{2}$. But we defined our coordinate $r$ precisely so that this be the case.

Therefore, we will assume in the following that (39) is exact. This requirement goes beyond SR, the EP, and the Newtonian limit. It is a partial replacement of Einstein's assumption that in vacuum the Ricci tensor $R_{i k}$ must be required to vanish. $\underline{\underline{48}}$ In fact, it can be shown to follow from the vanishing of the diagonal temporal component $R_{t t}$. Being a much weaker postulate than Einstein's, it gives us much less. The latter produces the exact Schwarzschild solution, the former fixes one of the functions $f$ and $g$ in terms of the other. So if we knew the expansion of $f(r)$ in powers of $G M / r c^{2}$, we could fully calculate the corresponding expansion of $g(r)$. But at this stage, we cannot even determine the coefficient $\beta_{2}$.

We conclude that just using the EP in trying to transcend SR and Newtonian gravity gives us the first-order term of the expansion of $f(r)$ in powers of $G M / r c^{2}$, but nothing more $\left(f(r)=1+\beta_{1} G M / r c^{2}\right.$ with $\left.\beta_{1}=-2\right)$, in accord with Refs. 21 and 22 . Our additional assumption about the validity of (39) beyond the weakfield limit produces a relationship between the coefficients of $f(r)$ and $g(r)$. In particular, setting $g(r)=$ $1 /\left(1-\alpha_{1} G M / r c^{2}-\ldots\right)$, we find

$$
\alpha_{1}=2\left(1-\beta_{2}\right)
$$

Essentially, our new postulate rests on the assumption that mass-energy is the only source of the gravitational field. It is not expected to hold in alternative theories of gravity, in which additional sources of the field are present. The Brans-Dicke theory, for example, has a scalar field leading to a variable effective gravitational constant. This bears some similarity to electrodynamics in a medium with varying dielectric coefficient, in which there would be apparent electrical charge distributions leading to a non-vanishing divergence of the electric field $(\nabla \cdot \boldsymbol{E} \neq 0)$. So the electrical field of a point charge in such a polarizable medium would not fall off as $1 / r^{2}$. Indeed, checking whether the postulate is satisfied in spherically 
symmetric solutions of the Brans-Dicke theory, we find that it is not, unless the scalar field is constant.

To obtain the exact result for $f(r)$ and $g(r)$, we would need a second postulate. While it is possible to generate a more plausible postulate than Tangherlini's second one, and while this postulate is not subject to Rindler's criticism, 28 both its physical justification and its practical use are somewhat more complex than that of the postulate about mass-energy conservation invoked so far (albeit still simpler than the field equations). Therefore, the presentation of that route to the exact Schwarzschild metric will be postponed to a different publication. It may not really be suited for the classroom at the early stage envisaged. Instead, we will take the point of view here, that if we cannot determine either $\alpha_{1}$ or $\beta_{2}$ from theoretical arguments, why not turn to experiments?

\section{THE PERIHELION PRECESSION OF MERCURY}

As it is assumed that the class has no prior knowledge on GR, we first need to provide an approach to the equations of motion of a particle in a given metric, i.e., the geodesic equations. This is done in the argument from Eqs. (51) to (53), based on the EP. If the material of this paper is used at a later stage in a GR course and the geodesic equations are already known, this argument may be skipped and reference can be made to the standard approach to the geodesic equations via the exact form of the Lagrangian given in (51).

We write the line element as

$$
\begin{aligned}
\mathrm{d} s^{2}= & -c^{2} \mathrm{~d} \tau^{2}=-\mathrm{e}^{2 \Phi(r) / c^{2}} c^{2} \mathrm{~d} t^{2}+\frac{1}{1-\alpha(r)} \mathrm{d} r^{2} \\
& +r^{2}\left(\mathrm{~d} \vartheta^{2}+\sin ^{2} \vartheta \mathrm{d} \varphi^{2}\right)
\end{aligned}
$$

with

$$
\begin{aligned}
\mathrm{e}^{2 \Phi(r) / c^{2}} & =1-\frac{2 \tilde{M}}{r}+\beta(r), \quad \tilde{M}=\frac{G M}{c^{2}}, \\
\beta(r) & =\beta_{2} \frac{\tilde{M}^{2}}{r^{2}}+\mathcal{O}\left(\frac{\tilde{M}^{3}}{r^{3}}\right), \\
\alpha(r) & =\alpha_{1} \frac{\tilde{M}}{r}+\alpha_{2} \frac{\tilde{M}^{2}}{r^{2}}+\mathcal{O}\left(\frac{\tilde{M}^{3}}{r^{3}}\right) .
\end{aligned}
$$

$M$ is assumed to be the mass of the sun and we treat a planet (Mercury) with mass $m$ moving in its gravitational field. Consider now the quantity (an overdot signifies a derivative with respect to proper time)

$$
\begin{aligned}
L= & \frac{m}{2}\left(\frac{\mathrm{d} s}{\mathrm{~d} \tau}\right)^{2}=\frac{m}{2}\left(\frac{1}{1-\alpha(r)} \dot{r}^{2}\right. \\
& \left.+r^{2} \dot{\vartheta}^{2}+r^{2} \sin ^{2} \vartheta \dot{\varphi}^{2}\right)-\frac{m}{2} \mathrm{e}^{2 \Phi(r) / c^{2}} c^{2} \dot{t}^{2} \\
\approx & \frac{m}{2}\left(\dot{r}^{2}+r^{2} \dot{\vartheta}^{2}+r^{2} \sin ^{2} \vartheta \dot{\varphi}^{2}\right)-\frac{m}{2} c^{2} \dot{t}^{2}-m \Phi(r) \dot{t}^{2} \\
\approx & \frac{m}{2}\left(r_{t}^{2}+r^{2} \vartheta_{t}^{2}+r^{2} \sin ^{2} \vartheta \varphi_{t}^{2}\right)-\frac{m}{2} c^{2}-m \Phi(r)
\end{aligned}
$$

$$
=T-V-\frac{m}{2} c^{2} .
$$

Herein, the first approximation uses the smallness of $\tilde{M} / r$ and the second takes advantage of the fact that a planet moves slowly in comparison with the speed of light, so $\dot{t} \approx 1$, i.e., global and proper time are almost the same, and derivatives with respect to the proper time may be replaced by derivatives with respect to $t$. The last line finally identifies the kinetic energy $T$ and the potential energy $V$ in the Newtonian limit. The NL of $L$ is a Newtonian Lagrangian, which suggests that $L$ itself might be a relativistic Lagrangian.

This conjecture can in fact be proven on the basis of the EP. What the principle tells us is that in a freely falling frame the local laws of motion are those of SR. Hence, in such a frame, obtainable by an appropriate local coordinate transformation from the global metric, a point mass moves along a straight line, which we can determine from local initial conditions, then transform back to obtain a piece of the trajectory in the global frame, which gives the initial conditions for the next (close-by) local frame to which we may transform. Continuing the procedure, we obtain a piecewise construction of the trajectory. A more elegant way rather than to construct pieces of the trajectory is to produce equations of motion in the global frame from those of the local frames and then find the full solution in the global frame directly. Now it is obvious that with the Minkowski line element the quantity

$$
L^{\prime}=\frac{m}{2}\left(\frac{\mathrm{d} s}{\mathrm{~d} \tau}\right)^{2}=\frac{m}{2}\left(\dot{X}^{2}+\dot{Y}^{2}+\dot{Z}^{2}-c^{2} \dot{T}^{2}\right)
$$

is a valid Lagrangian for special relativistic motion of a free particle. All coordinates are cyclic, so the equations of motion state that $T, X, Y, Z$ are linear functions of the proper time, which means that the four-velocity is constant. These are the correct equations of motion in SR. Transforming this Lagrangian back to the global frame is trivial, because both $\mathrm{d} s$ and $\mathrm{d} \tau$ are relativistic invariants (as is $m$ ), so the result of the transformation is $L$ of Eq. (51). Hence, we can derive the equations of motion in the metric from $L$.

Are there solutions with $\vartheta=\pi / 2=$ const. as in the Newtonian case? The equation of motion for $\vartheta$

$$
\frac{\mathrm{d}}{\mathrm{d} \tau} \frac{\partial L}{\partial \dot{\vartheta}}-\frac{\partial L}{\partial \vartheta}=\frac{\mathrm{d}}{\mathrm{d} \tau} m r^{2} \dot{\vartheta}-m r^{2} \sin \vartheta \cos \vartheta \dot{\varphi}^{2}=0
$$

is obviously solved by $\vartheta \equiv \frac{\pi}{2}$, so we may indeed restrict ourselves to motion in the equatorial plane.

Both $\varphi$ and $t$ are cyclic coordinates leading to conservation laws:

$$
\begin{aligned}
r^{2} \dot{\varphi} & =h=\text { const. } \\
\mathrm{e}^{2 \Phi / c^{2}} \dot{t}=k & =\text { const. }
\end{aligned}
$$

Equation (54) describes conservation of the component of angular momentum perpendicular to the equatorial plane, and Eq. (55) expresses energy conservation.

Finally, instead of writing down the Euler-Lagrange equation for $r$ (the many $r$ dependent terms would lead 
to a messy formula), we exploit the constancy of the Lagrangian itself $\AA^{49}$ to obtain another integral of the motion:

$$
-c^{2}=-\mathrm{e}^{2 \Phi / c^{2}} c^{2} \dot{t}^{2}+\frac{1}{1-\alpha(r)} \dot{r}^{2}+r^{2} \dot{\varphi}^{2} .
$$

Using (54) and (55), we can separate out an equation for the radial coordinate alone

$$
\frac{1}{1-\alpha(r)} \dot{r}^{2}+\frac{h^{2}}{r^{2}}+\left(1-k^{2} \mathrm{e}^{-2 \Phi / c^{2}}\right) c^{2}=0 .
$$

(The Newtonian limit of this equation is obtained by letting $c \rightarrow \infty$, which implies $k \rightarrow 1$ and leads to the familiar $\dot{r}^{2}+h^{2} / r^{2}-2 G M / r=0$.) We are interested in the spatial trajectory only, i.e., the function $r(\varphi)$, so we write $\dot{r}=\mathrm{d} r / \mathrm{d} \varphi \mathrm{d} \varphi / \mathrm{d} \tau=r^{\prime} \dot{\varphi}=r^{\prime} h / r^{2}$. It is then convenient to introduce the new variable $u(\varphi)=1 / r$, whence $u^{\prime}=$ $-r^{\prime} / r^{2}$. Using the expansions of $\alpha(r)$ and $\exp \left(2 \Phi(r) / c^{2}\right)$, multiplying the equation by $[1-\alpha(r(u))] / h^{2}$ and expanding all terms to second order in $\tilde{M} u$, we obtain after a rearrangement of terms:

$$
\begin{aligned}
u^{\prime 2} & +u^{2}-\alpha_{1} \tilde{M} u^{3}-\alpha_{2} \tilde{M}^{2} u^{4} \\
& -\frac{c^{2}}{h^{2}}\left(2 \tilde{M} u+k^{2}\left(4-2 \alpha_{1}-\beta_{2}\right) \tilde{M}^{2} u^{2}\right) \\
& +\frac{c^{2}}{h^{2}}\left(1-k^{2}\right)\left(1+\left(2-\alpha_{1}\right) \tilde{M} u-\alpha_{2} \tilde{M}^{2} u^{2}\right)=0 .
\end{aligned}
$$

In order to simplify this equation, we consider the sizes of its terms. The leading order terms are $u^{\prime 2}+u^{2}-$ $2 c^{2} \tilde{M} u / h^{2}+c^{2}\left(1-k^{2}\right) / h^{2}$. While $\tilde{M} u$ is very small $\underline{50}$ the first term linear in $u$ is multiplied by $c^{2}$, a large factor. This is the reason why we have to take into account the $u^{2}$ term in the first parentheses. However, we may drop the $\alpha_{2}$ term in the first line and also the one multiplied by $c^{2}\left(1-k^{2}\right) / h^{2}$, because in this term the large factor $c^{2}$ is compensated by the small factor $1-k^{2}$. To see this, let us estimate $k$, by evaluating Eq. (57) at the perihelion, where $\dot{r}=0$, and taking the NL. The Newtonian value for the minimum distance of the planet to the center of motion is $r_{\min }=h^{2} /(G M(1+e))$, where $e$ is the eccentricity and $h$ twice the areal velocity, referred to Newtonian time instead of proper time. We find

$$
k^{2}-1=-\frac{G M}{c^{2} a},
$$

where $a=r_{\min } /(1-e)$ is the semi-major axis of the orbital ellipse of the planet considered. The result is twice the orbital energy of the planet divided by $m c^{2}$, a very small quantity indeed.

Having justified the neglect of the $\alpha_{2}$ terms in (58), we take the derivative with respect to $\varphi$ (to obtain a linear lowest-order equation), and get, after dividing off the common factor $2 u^{\prime}$

$$
\begin{aligned}
u^{\prime \prime}+u= & \frac{G M}{h^{2}}+\tilde{M}\left(\frac{3}{2} \alpha_{1} u^{2}+k^{2}\left(4-2 \alpha_{1}-\beta_{2}\right) \frac{G M}{h^{2}} u\right. \\
& \left.-\frac{c^{2}}{h^{2}}\left(1-k^{2}\right)\left(1-\frac{\alpha_{1}}{2}\right)\right) .
\end{aligned}
$$

Herein, we may consider the term multiplied by $\tilde{M}$ a small perturbation, first solve the equation with $\tilde{M}$ set equal to zero and then correct the result using perturbation theory. The lowest-order equation is $u^{\prime \prime}+u=$ $G M / h^{2}$ and it is solved by

$$
u_{0}=\frac{1}{r_{0}}=\frac{G M}{h^{2}}(1+e \cos \phi),
$$

the well-known Newtonian result. The eccentricity $e$ is one of the integration constants. Another would be the angle $\varphi_{0}$ between the semi-major axis and the $x$ axis, which has been absorbed into a redefinition of the $x$ axis, so the perihelion is at $\varphi=0$. The results for $r_{\min }$ and $a$ used in deriving (59) immediately follow from (61). Note that only the term $\propto u^{2}$ has to be treated within perturbation theory. Without it, Eq. (60) would be solvable exactly. Because we still would have to treat one term perturbatively, we might as well consider all terms multiplied by $\tilde{M}$ perturbations.

Now we iterate the equation, inserting $u_{0}$ on the righthand side, to obtain the first-order correction:

$$
\begin{aligned}
& u^{\prime \prime}+u=\frac{G M}{h^{2}}+\tilde{M} \frac{G^{2} M^{2}}{h^{4}}\left[\frac { 3 } { 2 } \alpha _ { 1 } \left(1+\frac{e^{2}}{2}+2 e \cos \varphi\right.\right. \\
&+\left.\left.\frac{e^{2}}{2} \cos 2 \varphi\right)+k^{2}\left(4-2 \alpha_{1}-\beta_{2}\right)(1+e \cos \varphi)\right] \\
&-\tilde{M} \frac{c^{2}}{h^{2}}\left(1-k^{2}\right)\left(1-\frac{\alpha_{1}}{2}\right) .
\end{aligned}
$$

This is the equation of motion of a driven harmonic oscillator with resonant terms on the right-hand side (the terms $\propto \cos \varphi$ ). A straightforward treatment would lead to self-amplifying solutions, destroying the applicability of perturbation theory. Therefore, we use a slightly more sophisticated approach, the Poincaré-Lindstedt method, in which the argument of the solution is considered a function of the perturbation, too. Restricting ourselves to the lowest-order scheme, we write $u(\varphi)=\tilde{u}((1+\varepsilon) \varphi)$, with $\varepsilon$ being proportional to the small parameter $\tilde{M}$. We then have $u^{\prime \prime}+u=(1+\varepsilon)^{2} \tilde{u}^{\prime \prime}+\tilde{u} \approx(1+2 \varepsilon) \tilde{u}^{\prime \prime}+\tilde{u}$ and setting $\tilde{u}=\tilde{u}_{0}+\tilde{M} \tilde{u}_{1}$, we obtain $\tilde{u}_{0}^{\prime \prime}+2 \varepsilon \tilde{u}_{0}^{\prime \prime}+\tilde{M} \tilde{u}_{1}^{\prime \prime}+\tilde{u}_{0}+\tilde{M} \tilde{u}_{1}$ on the left-hand side of (62). Since $\tilde{u}_{0}=u_{0}$, the term multiplied by $\varepsilon$ is proportional to $\cos \varphi$ and by an appropriate choice of $\varepsilon$, we may cancel the secular terms on the right-hand side. Then $\tilde{u}_{1}$ satisfies an equation of the type $\tilde{u}_{1}^{\prime \prime}+\tilde{u}_{1}=A+B \cos 2 \varphi$, but we are not particularly interested in solving it, as the information about the perihelion precession is in the modified periodicity of the solution, determined already by the value of $\varepsilon$. The new period is $P=2 \pi /(1+\varepsilon) \approx 2 \pi(1-\varepsilon)$, hence the perihelion shift $\Delta P$ per period is

$$
\Delta P=-2 \pi \varepsilon=\pi \tilde{M} \frac{G M}{h^{2}}\left(\alpha_{1}-\beta_{2}+4\right),
$$

where we have replaced $k^{2}$ by 1 . The formula may be recast in terms of more convenient quantities. $h$ is twice the areal velocity, hence in the approximation of a Kepler ellipse

$$
h=2 \frac{\pi a b}{T}=2 \frac{\pi a^{2} \sqrt{1-e^{2}}}{T},
$$


with $T$ its orbital period, and from Kepler's third law

$$
T^{2}=\frac{4 \pi^{2} a^{3}}{G M},
$$

we obtain an expression for $G M$. Combining the two results, we have $(G M)^{2} / h^{2}=4 \pi^{2} a^{2} /\left(T^{2}\left(1-e^{2}\right)\right)$ and find

$$
\Delta P=\frac{4 \pi^{3} a^{2}}{c^{2} T^{2}\left(1-e^{2}\right)}\left(\alpha_{1}-\beta_{2}+4\right) .
$$

The annual perihelion shift $\Delta P_{a}$ is obtained from this by multiplying with $T_{\text {earth }} / T$, which is a factor of 4.152 for Mercury, having an orbital period of $87.969 \mathrm{~d}$. Plugging in numbers, we get $\Delta P_{a}=3.4730 \times 10^{-7}\left(\alpha_{1}-\beta_{2}+4\right)$. This is the result in radians. To convert it to arcseconds, we note that $1^{\prime \prime}=2 \pi / 360 / 3600 \mathrm{rad}=4.8481 \times 10^{-6} \mathrm{rad}$. Then we have $\Delta P_{a}=0.0716^{\prime \prime} \times\left(\alpha_{1}-\beta_{2}+4\right)$. Experimental measurements give $\Delta P_{a}=0.4298^{\prime \prime}, \underline{51}$ from which we infer

$$
\alpha_{1}-\beta_{2}+4 \approx \frac{0.4298^{\prime \prime}}{0.0716^{\prime \prime}}=6.003 .
$$

This immediately leads to the conjecture

$$
\alpha_{1}-\beta_{2}=2 .
$$

Together with $\alpha_{1}=2\left(1-\beta_{2}\right)$ from Sec. III we end up with

$$
\alpha_{1}=2, \quad \beta_{2}=0 .
$$

Therefore, we have now inferred the Schwarzschild metric (42) to second-order accuracy in the small parameter $G M / r c^{2}$ for the coefficient $g_{t t}=f(r)$ and to firstorder accuracy for $g_{r r}=g(r)$, which is known as the first parameterized post-Newtonian approximation (PPN) $\underline{52}$ Clearly, the two parameters determined in this section from experimental information would be known only with finite precision. They could not be claimed to be exact without the benefits of the field theory.

We conclude that Einstein might indeed have used a similar approach in 1911 and would then have been able to correctly predict gravitational light deflection by the sun five years earlier than he actually did. Also, he might have found the Schwarzschild solution before Schwarzschild $\underline{53}$ and Droste $\underline{\underline{54}}$

As it turns out, experimental information on light deflection gives much simpler access to the coefficient $\alpha_{1}$ than perihelion precession data. So let us turn to a brief analysis of the behavior of light in the gravitational field of a spherically symmetric mass distribution.

Unfortunately, this experimental informaton was not available before 1919 and then only with low accuracy 55

\section{LIGHT DEFLECTION}

Again, if the geodesic equations are supposed known, part of this section can be skipped. Even then, the use of isotropic Schwarzschild coordinates suggested here is favorable, as it leads to simpler equations and allows one to repeat the argument about the factor of 2 between the EP prediction and the full calculation, given below. Given the equations for null geodesics, one may eliminate the affine parameter, determine the energy constant $k$ from the limit $r \rightarrow \infty$ and introduce the variable $u=b / \rho$, which produces Eq. (79).

Assuming instead no prior knowledge of the geodesic equations, it may be argued that just as the principle of least action governs the motion of particles and gives us the equations of motion, once we know the Lagrangian, Fermat's principle governs the paths of light and gives us their equations, once we know the index of refraction or, equivalently, the speed of light. Both principles have the advantage of being coordinate free, so we may expect them to work in curved spacetime without problems. It should not matter whether a given coordinate system describes a patch of flat or of curved spacetime.

Let us therefore put Fermat's principle to use in describing light deflection. It is obvious that the coordinate speed of light, obtained from the line element by setting $\mathrm{d} s^{2}=0$, varies in a metric such as (26). We may interpret this in terms of a variable refractive index $n$, and then require, in order to calculate the path of light

$$
\delta S_{F}=0 \quad \text { for } \quad S_{F}=\frac{1}{c} \int \mathrm{d} l n(l)=\int \frac{\mathrm{d} l}{c(l)},
$$

where $\mathrm{d} l$ is the (coordinate) length element of the path of the light ray and $c(l)$ is the local speed of light. The endpoints of the path are supposed to be fixed. Hereafter, I will slightly abuse notation in taking $c$ for the universal speed of light and $c(x)$ for the coordinate speed of light at some point $x$.

When written in the form (70), the principle requires $n(x)$ and hence $c(x)$ to be a scalar function, i.e., the velocity of light should be isotropic. This is clearly not true for the general metric (26). The velocity of light in the radial direction is given by $c_{r}(r)=\mathrm{d} r / \mathrm{d} t=\sqrt{f(r)} c / \sqrt{g(r)}$ (setting $\mathrm{d} s, \mathrm{~d} \vartheta$ and $\mathrm{d} \varphi$ equal to zero), whereas the transverse speed of light is $c_{t}(r)=\sqrt{f(r)} c$ (setting $\mathrm{d} s$ and $\mathrm{d} r$ equal to zero).

While it is possible to phrase Fermat's principle for an-

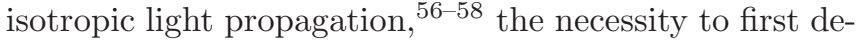
rive this unfamiliar formulation makes it unattractive for work in class. Instead, we rewrite our metric to spatially isotropic form. This can be achieved via introduction of a new radial coordinate $\rho$ as follows: set $r=r(\rho)$ and require

$$
g(r) r^{\prime}(\rho)^{2}=\frac{r^{2}}{\rho^{2}} .
$$

Solving this differential equation for $r$ gives a metric with spatial part $g(r(\rho)) r^{\prime}(\rho)^{2}\left(\mathrm{~d} \rho^{2}+\rho^{2} \mathrm{~d} \vartheta^{2}+\rho^{2} \sin ^{2} \vartheta \mathrm{d} \varphi^{2}\right)$. For the metric (47) with $\alpha(r)$ approximated by the first term of (50), this procedure leads to

$$
r=\rho\left(1+\frac{\alpha_{1} \tilde{M}}{4 \rho}\right)^{2},
$$


where the integration constant has been chosen so that far from the central mass both coordinates become equal to each other. In the new coordinates, the spacetime line element takes the form

$$
\mathrm{d} s^{2}=-k_{1}^{2} c^{2} \mathrm{~d} t^{2}+k_{2}^{2} \mathrm{~d} l^{2},
$$

where

$$
\begin{aligned}
\mathrm{d} l^{2} & =\mathrm{d} \rho^{2}+\rho^{2} \mathrm{~d} \vartheta^{2}+\rho^{2} \sin ^{2} \vartheta \mathrm{d} \varphi^{2}, \\
k_{1} & =\left(1-\frac{2 \tilde{M}}{\rho}\right)^{1 / 2}+\mathcal{O}\left(\frac{\tilde{M}^{2}}{\rho^{2}}\right), \\
k_{2} & =\left(1+\frac{\alpha_{1} \tilde{M}}{4 \rho}\right)^{2}+\mathcal{O}\left(\frac{\tilde{M}^{2}}{\rho^{2}}\right) .
\end{aligned}
$$

The local coordinate speed of light is then given by

$$
c(\rho)=\frac{k_{1}}{k_{2}} c=\left(1-\frac{\left(2+\alpha_{1}\right) \tilde{M}}{2 \rho}\right) c+\mathcal{O}\left(\frac{\tilde{M}^{2}}{\rho^{2}}\right) .
$$

For symmetry reasons, we expect the path of a light ray in the equatorial plane $\vartheta=\pi / 2$ to remain in that plane, so we can drop the $\mathrm{d} \vartheta$ contribution to the spatial coordinate line element $\mathrm{d} l$. We are then left with the task to minimize

$$
S_{F}=\int \frac{\sqrt{\mathrm{d} \rho^{2}+\rho^{2} \mathrm{~d} \varphi^{2}}}{c(\rho)}=\int \frac{\sqrt{\rho^{\prime}(\varphi)^{2}+\rho(\varphi)^{2}}}{c(\rho(\varphi))} \mathrm{d} \varphi .
$$

Taking the integrand to be a function $s_{F}\left(\rho^{\prime}(\varphi), \rho(\varphi), \varphi\right)$, we note it does not depend on $\varphi$ explicitly, so the "Hamiltonian"

$$
\rho^{\prime} \frac{\partial s_{F}}{\partial \rho^{\prime}}-s_{F}
$$

is constant. Naming the constant $-b / c$, we find

$$
\frac{\rho^{2}}{\sqrt{\rho^{\prime 2}+\rho^{2}}}=b \frac{c(\rho)}{c} .
$$

Solving this algebraically for $\rho^{\prime}$ and substituting $u=b / \rho$, we obtain

$$
u^{\prime 2}+u^{2}=\frac{c^{2}}{c(\rho(u))^{2}}=1+\frac{\left(2+\alpha_{1}\right) \tilde{M} u}{b}+\mathcal{O}\left(\frac{\tilde{M}^{2}}{b^{2}}\right)
$$

Differentiating with respect to $\varphi$ and dividing through by $2 u^{\prime}$, we end up with an extremely simple equation

$$
u^{\prime \prime}+u=\frac{\left(2+\alpha_{1}\right) \tilde{M}}{2 b} .
$$

This is a shifted harmonic oscillator. Rewritten in terms of $\rho$, the solution reads

$$
\frac{b}{\rho}=\sin \left(\varphi-\varphi_{0}\right)+\frac{\left(2+\alpha_{1}\right) \tilde{M}}{2 b} .
$$

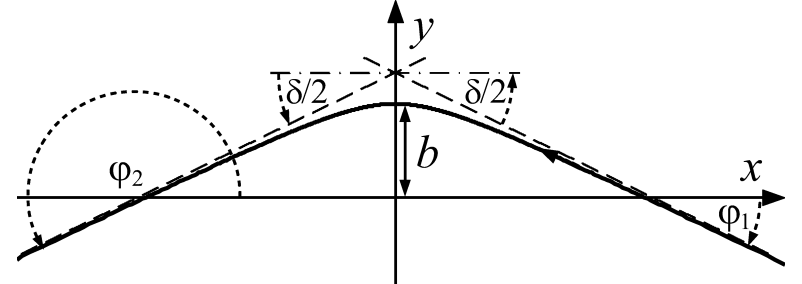

FIG. 2. Pictorial representation of the trajectory of a photon and its orientation in the coordinate system with $x=\rho \cos \varphi$, $y=\rho \sin \varphi$. Small angles are generously exaggerated in size.

From this equation, describing a hyperbola, we can read off the deflection angle. For convenience, we set $\varphi_{0}=0$, which gives the hyperbola the orientation shown in Fig. 2 (assuming $b>0$ ).

For $\rho \rightarrow \infty$, given the smallness of the second term on the right-hand side of Eq. [81], the argument of the sine must go to $\varphi_{1}=-\left(1+\alpha_{1} / 2\right) \tilde{M} / b$ for $x>0$ and to $\varphi_{2}=\pi+\left(1+\alpha_{1} / 2\right) \tilde{M} / b$ for $x<0$. The deflection angle then is

$$
\delta \equiv \varphi_{2}-\pi-\varphi_{1}=\left(1+\frac{\alpha_{1}}{2}\right) \frac{2 G M}{b c^{2}} .
$$

With $\alpha_{1}=2$, Einstein's 1916 result $\delta=4 G M /\left(b c^{2}\right)$ is recovered, corresponding to an angle of 1.75 " for a light ray that grazes the surface of the sun (i.e., when $b$ is equal to the radius of the sun).

The utility of isotropic coordinates in the calculation of this effect may be underlined by a comment regarding the factor of 2 between the correct (first-order) result and Einstein's original result. In his 1911 paper, Einstein derived the speed of light from the equivalence principle alone, which means that he replaced the local gravitational field by a patch of an inertial field with a value $g_{t t}$ adapted to the true field, but effectively with $g_{r r}=1$. He therefore obtained

$$
c(r)=\left(1+\frac{\Phi(r)}{c^{2}}\right) c=\left(1-\frac{\tilde{M}}{r}\right) c .
$$

Plugging this velocity into Fermat's principle, we get a formula in which the deviation of the coordinate speed of light from the vacuum speed of light is formally half the value of the deviation in the isotropic Schwarzschild metric, if we rename $r$ to $\rho$ (compare with Eq. (75) for $\left.\alpha_{1}=2\right)$. Since the angular deflection is so small that it is easily captured by perturbation theory, the final result must be linear in this deviation (from the zeroth-order straight-line solution), so the angle of deflection obtained in the full theory must be a factor of 2 larger than the one obtained by the EP only. No argument of comparable simplicity is available when comparing a calculation based on the EP with the full theory using the original Schwarzschild metric. In that metric, the speed of light is anisotropic, agreeing with the prediction from the EP for transverse light rays and differing from it for radial ones. (Far from the sun, the ray is essentially radial.)

Note that if we assume the experimental result on light deflection to be available, we can deduce the value of $\alpha_{1}$ 
without the need to require that (39) is valid beyond the lowest order in $\tilde{M}$. Hence the experiment allows us to determine this coefficient without any second-order knowledge of $f(r)$, whereas we need this information, if we want to obtain $\alpha_{1}$ from the perihelion precession. How does this come about?

Consider the complete Lagrangian of Eq. (51). In it, the term $\propto \dot{r}^{2}$ is much smaller than the term $\propto c^{2} \dot{t}^{2}$, because the velocity $\dot{r}$ of a planet is much smaller than $c$. So in order to obtain an accurate estimate of the small quantity $\alpha_{1} \tilde{M} / r$ appearing as a factor in the former term, we have to know much smaller factors of the latter term, i.e., we have to calculate $f(r)$ to second-order precision. The case of light bending is different. Here the $\mathrm{d} r^{2}$ and $\mathrm{d} t^{2}$ terms of the line element are the same order of magnitude, because for light $\mathrm{d} r / \mathrm{d} t$ is on the order of $c$. Thus, to determine $g_{r r}$ accurate to first order from experiment, it is sufficient to know $g_{t t}$ to first order.

\section{CONCLUSIONS}

It is well-known that out of the three classical tests of general relativity, the gravitational redshift, essentially explicable in terms of the EP, does not probe the field equations, whereas light deflection in a gravitational field and the perihelion precession do depend on them. The same is true for the fourth test conceived later, the Shapiro delay, not discussed in detail here.

We have explored in this paper, how far some simple ideas, not exploiting the full field theory, may carry us in determining a usable weak-field approximation of the metric outside a spherically symmetric mass distribution. This amounts to the approximate construction of no more than two radial functions. As it was not expected that these ideas would generate enough information to predict spacetime curvature quantitatively, we were willing to accept one adjustable parameter to emerge from one of the three experiments probing the field equations, which would then, hopefully, allow us to make quantitative predictions of the other two. In part, this was motivated by the increase in credibility that a "physics first" approach to GR would gain, if the metric employed to derive predictions could be justified without use of the field equations.
It turned out that this program is feasible but that the two experiments considered need different levels of additional information.

In the case of the perihelion precession, to progress at all we had to make an assumption about the range of validity of the force law (39), because two unknown coefficients of the metric are needed in a lowest-order PPN description. Given that new assumption and a measurement of the perihelion precession, we get the metric with sufficient accuracy to predict both the outcome of the light bending experiment and the Shapiro effect quantitatively 59

In the case of light bending by the sun, only one parameter is missing in the metric as far as it is determined by the EP and the NL. A measurement of light deflection fixes this parameter. With the metric so obtained, the Shapiro delay could be predicted quantitatively without any additional postulate on the force law. However, the same metric would be insufficient to quantitatively predict the perihelion precession of Mercury. Assuming the force law to be accurate beyond lowest order, enough information can be gathered.

In regard to research, our results may be considered not overly interesting, since the exact solution of the field equations is known. But they might be of some use in the classroom. One of the calculations from Secs. IV and $\mathrm{V}$ can be discussed during a course, the other may be assigned as a homework. Physical understanding of the spherically symmetric system would be greatly improved. The experience that our attempt at a simplification, avoiding the field equations, involves moderately extensive calculations at least in the case of the perihelion precession, will help students to appreciate the introduction of the field equations later, which allow, with a little more effort, to obtain the exact form of the spherically symmetric static metric.

Acknowledgment I am grateful to Robert Shuler from NASA, Johnson Space Center, who set me on track and largely inspired this work. His idea was to start from the result for the measurement of forces with a tether in the Schwarzschild metric and to invert the procedure in order to obtain the metric from the force law. When this did not quite work out, he suggested to use experimental results to get the missing information.
* Klaus.Kassner@ovgu.de

1 T. A. Debs and M. L. G. Redhead, "The twin "paradox" and the conventionality of simultaneity," Am. J. Phys. 64, 384-392 (1996)

2 M. B. Cranor, E. M. Heider, and R. H. Price, "A circular twin paradox," Am. J. Phys. 68, 1016-1020 (1999)

3 J. P. van der Weele and J. H. Snoeijer, "Beyond the PoleBarn Paradox: How the Pole is Caught," Nonlinear Phenomena in Complex Systems 10, 271-277 (2007)

${ }^{4}$ Moses Fayngold, Special Relativity and Motions Faster than Light (Wiley-VCH Verlag GmbH, Weinheim, Germany, 2002)

5 J. W. Butler, "The Lewis-Tolman Lever Paradox," Am. J.
Phys. 38, 360-368 (1970)

${ }^{6}$ E. M. Dewan and M. J. Beran, "Note on stress effects due to relativistic contraction," Am. J. Phys. 27, 517-518 (1959)

7 J. S. Bell, "How to teach special relativity," in Speakable and unspeakable in quantum mechanics (Cambridge University Press, 1993) pp. 67-80, first published in Progress in Scientific Culture 1, 1976

8 P. Ehrenfest, "Gleichförmige Rotation starrer Körper und Relativitätstheorie," Phys. Zeitschrift 10, 918-918 (1909)

$9 \varnothing$. Grøn, "Special-Relativistic Resolution of Ehrenfest's Paradox: Comments on Some Recent Statements by T. E. Phipps, Jr.." Found. Phys. 11, 623-631 (1981) 
${ }^{10}$ K. Kassner, "Spatial geometry of the rotating disk and its non-rotating counterpart," Am. J. Phys. 80, 772-781 (2012)

11 C. W. Berenda, "The Problem of the Rotating Disk," Phys. Rev. 62, 280-290 (1942)

$12 \varnothing$. Grøn, "Relativistic description of a rotating disk," Am. J. Phys. 43, 869-876 (1975)

13 J. B. Hartle, "General relativity in the undergraduate physics curriculum," Am. J. Phys. 74, 14-21 (2006)

14 N. Christensen and T. Moore, "Teaching general relativity to undergraduates," Physics Today 65(6), 41-47 (2012)

15 A. Sommerfeld, Electrodynamics. Lectures on Theoretical Physics, Vol. III (Academic Press, New York, 1952)

16 L. I. Schiff, "On Experimental Tests of the General Theory of Relativity," Am. J. Phys. 28, 340-343 (1960)

17 W. M. Sacks and J. A. Ball, "Simple derivations of the Schwarzschild metric," Am. J. Phys. 36, 240-245 (1968)

${ }^{18}$ W. Rindler, "Counterexample to the Lenz-Schiff Argument," Am. J. Phys. 36, 540-544 (1968)

19 P. Rowlands, "A simple approach to the experimental consequences of general relativity," Physics Education 32, 4955 (1997)

20 R. R. Cuzinatto, B. M. Pimental, and P. J. Pompeia, "Schwarzschild and de Sitter solutions from the argument by Lenz and Sommerfeld," Am. J. Phys. 79, 662-667 (2011)

21 A. Schild, "Equivalence Principle and Red-Shift Measurements," Am. J. Phys. 28, 778-780 (1960)

22 R. P. Gruber, R. H. Price, S. M. Matthews, W. R. Cordwell, and L. F. Wagner, "The impossibility of a simple derivation of the Schwarzschild metric," Am. J. Phys. 56, 265-269 (1988)

${ }^{23} G$ is Newton's gravitational constant, $c$ the speed of light, $M$ the mass at the center of gravity and $r$ the radial coordinate at which the metric is considered.

24 To the uninitiated, these are qualitatively different axioms with very different levels of plausibility.

${ }^{25}$ Here, we are so accustomed to their equivalence that we do not even see them as different postulates, which formally they are.

${ }^{26}$ Hence, the postulate(s) should be derivable from the field equations but need not imply their general form.

27 F. R. Tangherlini, "Postulational Approach to Schwarzschild's Exterior Solution with Application to a Class of Interior Solutions," Nuovo Cimento 25, 1081-1105 (1962)

28 W. Rindler, "Counterexample to the Tangherlini Argument," Am. J. Phys. 37, 72-73 (1969)

29 A. Einstein, "Über den Einfluß der Schwerkraft auf die Ausbreitung des Lichtes," Ann. Phys. (Berlin) 340, 898908 (1911)

30 J. G. v. Soldner, "Über die Ablenkung eines Lichtstrahls von seiner geradlinigen Bewegung, durch die Attraktion eines Weltkörpers, an welchem er nahe vorbei geht," Berliner Astronomisches Jahrbuch, 161-172 (1804)

31 In the present context, by a true experiment as opposed to a thought one I mean an experiment the outcome of which cannot be predicted quantitatively based on theories known before GR.

32 I. I. Shapiro, "Fourth Test of General Relativity," Phys. Rev. Lett. 13, 789-791 (1964)

33 M. Born, "Die Theorie des starren Elektrons in der Kinematik des Relativitätsprinzips," Ann. Phys. (Berlin) 335, 1-56 (1909)

34 The symmetry properties of the resulting formulas ensure that the distance is then constant in the frame of $\mathrm{O}_{2}$ as well.
35 In fact, there could be a term of the form $H(\tilde{x}) \mathrm{d} t \mathrm{~d} \tilde{x}$, but this can be transformed away by a resynchronization transformation of time, as is briefly discussed for the spherically symmetric metric in Sec. III

$36 \varnothing$. Grøn, "Covariant formulation of Hooke's law," Am. J. Phys. 49, 28-30 (1981)

37 Since real tethers always violate this condition to some extent, we might contemplate various contraptions to realize it to arbitrary precision. One possibility would be to make the tether a chain consisting of very short links and to monitor its local length changes by an appropriate device attached to it. Each time a local length unit is stretched by the extension of one link, the device removes one link and joins the separated parts of the chain; each time a local lengh unit is compressed by the length of a link, a link is inserted by the device.

38 A similar statement applies to the attribute massless, which is also not realizable exactly.

39 To make sure that we never end up with photons whose energy is insufficient to create an electron-positron pair, the process could be started with such a pair at rest near the upper observer, so the initial energy would be $2 E_{0}$ with $E_{0}=E_{e}(v=0)=m c^{2}$. After the electron and positron are sent down to the lower observer, they will have acquired kinetic energy, so that their total energy remains $2 E_{0}$ (the rest energy now being $2 \beta E_{0}$ ) from the point of view of the upper observer. Conversion to photons will transform the total energy into $2 \alpha E_{0} / \beta$ and the photons arriving back at the upper observer will then have a frequency $\nu=\alpha \nu_{0} / \beta$, where $h \nu_{0}=E_{0}$. Now either the energy $2(\alpha / \beta-1) E_{0}$ can be extracted and the remainder used to create an electronpositron pair at rest, to be sent down and starting an identical repetition of the cycle. Or else an electron-positron pair is created directly with some kinetic energy. Repetition of the cycle will then gain another factor $\alpha / \beta$ in energy and energy extraction may be delayed until a sufficiently large chunk of energy is available to be immediately useful.

40 It is different for observers at different $x_{i}$ values, as $f\left(x_{0}\right)$ in (23) gets replaced by $f\left(x_{i}\right)$. Hence, $F=-m a\left(x_{i}\right)$ for observer $O_{i}$.

$41 \varnothing$. Grøn, "Acceleration and weight of extended bodies in the theory of relativity," Am. J. Phys. 45, 65-79 (1977)

42 G. Muñoz and P. Jones, "The equivalence principle, uniformly accelerated frames, and the uniform gravitational field," Am. J. Phys. 78, 377-383 (2010)

43 The expression $-f(r) c^{2} \mathrm{~d} \tilde{t}^{2}+h(r) \mathrm{d} \tilde{t} \mathrm{~d} r+\tilde{g}(r) \mathrm{d} r^{2}$ changes, on setting $\mathrm{d} \tilde{t}=\mathrm{d} t+w^{\prime}(r) \mathrm{d} r$, into $-f(r) c^{2} \mathrm{~d} t^{2}+(h(r)-$ $\left.2 f(r) c^{2} w^{\prime}(r)\right) \mathrm{d} t \mathrm{~d} r+\left(\tilde{g}(r)-f(r) c^{2} w^{\prime}(r)^{2}+h(r) w^{\prime}(r)\right) \mathrm{d} r^{2}$. Requiring $w^{\prime}(r)=h(r) /\left(2 f(r) c^{2}\right)$, we can eliminate the off-diagonal term, and the prefactor of $\mathrm{d} r^{2}$ is simply renamed into $g(r)$.

44 The first was Einstein's way of using the EP, whereas C. Will $1^{60}$ is a strong proponent of the second way.

45 The approximations used in applying the equivalence principle become exact in the limit of an infinitesimal distance between the two observers exchanging a light signal. Therefore, the differential equation (33) is exact.

46 Equation (40) contains two approximations. The first is indicated by the $\approx$ sign, the second is the replacement of $\Phi(r)$ by its Newtonian limit. These two approximations happen to cancel each other to give the exact $f(r)$.

47 An observer at finite $r_{0}$ will obtain a force field that has the same radial behaviour but is larger by a constant factor $1 / \sqrt{f\left(r_{0}\right)}$. Moreover, he will have to use poles instead of tethers to measure the field at $r>r_{0}$.

48 A. Einstein, "Die Grundlage der allgemeinen Relativitäts- 
theorie," Ann. Phys. (Berlin) 354, 769-822 (1916), English Translation in The Principle of Relativity (Methuen, 1923, Reprinted by Dover Publications, New York, 1952), pp. $109-164$.

49 That the Lagrangian is constant here is a consequence of its definition in terms of invariants. We have $\mathrm{d} s^{2}=-c^{2} \mathrm{~d} \tau^{2}$, hence $L=-m c^{2} / 2$. On the other hand, the constancy of the Hamiltonian in classical mechanics follows from a conservation law, energy conservation, implied by invariance under time translations. But the Hamiltonian itself is not an invariant under arbitrary coordinate tranfsormations. In classical mechanics, the Lagrangian of a relativistic free particle is $L=-m c^{2} \sqrt{1-v^{2} / c^{2}}$, which is not a constant. The difference comes from the fact that there the action integral is $\int L \mathrm{~d} t$, where $t$ is the time of some inertial system, whereas here we define it as $\int L \mathrm{~d} \tau$, and the relationship between proper time $\tau$ and $t$ is, of course, $\mathrm{d} \tau=\sqrt{1-v^{2} / c^{2}} \mathrm{~d} t$.

50 An estimate for $\tilde{M} u$ is provided by $G M / c^{2} a \approx 2.55 \times 10^{-8}$, where $a \approx 57.9 \times 10^{9} \mathrm{~m}$ is the semi-major axis of Mercury's orbit.

51 English Wikipedia, "Tests of general Relativity," http://en.wikipedia.org/wiki/Tests_of_general_relativit

52 C. W. Misner, K. S. Thorne, and J. A. Wheeler, Gravitation (W. H. Freeman, New York, 1973)

${ }^{53}$ K. Schwarzschild, "Über das Gravitationsfeld eines
Massenpunktes nach der Einsteinschen Theorie," in Sitzungsberichte der Königlich-Preussischen Akademie der Wissenschaften (Reimer, Berlin, 1916) pp. 189-196, English translation: On the Gravitational Field of a Mass Point According to Einstein's Theory S. Antoci and A. Loinger, arXiv:physics/9905030 1

54 J. Droste, "The field of a single centre in Einstein's theory of gravitation, and the motion of a particle in that field," Ned. Acad. Wet., SA 19, 197-215 (1917)

${ }^{55}$ F. W. Dyson, A. S. Eddington, and C. R. Davidson, "A Determination of the Deflection of Light by the Sun's Gravitational Field, from Observations Made at the Total Eclipse of May 29, 1919," Philos. Trans. R. Soc. London A 220, 291 (1920)

${ }^{56}$ H. Weyl, "Zur Gravitationstheorie," Ann. Phys. (Berlin) 359, 117-145 (1917)

57 I. Kovner, "Fermat principle in arbitrary graviational fields," Astrophys. J. 351, 114-120 (1990)

58 R. Nityananda and J. Samuel, "Fermat's principle in general relativity," Phys. Rev. D 45, 3862-3864 (1992)

${ }^{59}$ I have not discussed the Shapiro delay here, but it is clear that its quantitative description is possible as soon as we ty have a metric that accurately describes gravitational light deflection.

60 C. M. Will, Was Einstein Right? Putting General Relativity to the test, 2nd ed. (Oxford University Press, Oxford, 1995) 